\title{
Research and Application of Modular System for Assembly-Type Barrier-free Facilities
}

\author{
Haihua Su \\ School of Architectural Engineering, Jiangsu Open University \\ suhh@jsou.cn
}

Keywords: Assembly Accessibility, Modular System, Assembly Accessibility Ramp, Design Ideas

\begin{abstract}
At present, prefabricated barrier-free facilities have been applied in various industries, playing a huge role, especially in urban road construction, the construction of modular system of prefabricated barrier-free facilities is quite deep in place. This paper discusses the modularization system of prefabricated barrier-free ramp facilities in urban road construction. Its construction concept is advanced and standardized, which can bring great convenience to traffic users. On the basis of discussing the present situation of modular system design and construction of assembly barrier-free ramp, the paper also studies its design and application idea in depth, hoping to improve its application efficiency.
\end{abstract}

\section{Introduction}

Under the new situation, the precast concrete members have solved the traditional difficult problem of steel structure. In the urban modern traffic road facilities system, the barrier-free ramp is essential, its construction idea is advanced and the standard rules are perfect, which can help the traffic users to pass through the road smoothly. At present, many cities are equipped with a certain number of prefabricated barrier-free facilities, forming a functional and even intelligent transportation modular system. On the one hand, it ensures the quality of traffic road construction, on the other hand, it also reduces the cost of municipal engineering construction, and kills two birds with one stone.

\section{Basic Overview of Accessible Ramps}

Modern urban road engineering construction is fast and the technology is widely used. At the same time, it also pays special attention to the flexible application of the people-oriented idea, emphasizing the safety, smooth, convenient and orderly traffic road construction to meet the travel needs of urban citizens. However, from the traditional urban road construction point of view, the general use of cross-sectional layout design, that is, non-motorized lanes and pedestrians and other slow traffic completely set on the side stone, that is, to trust a certain independent road rights. At the same time, it is also necessary to take into account the rigid needs of the disabled, and combine the impact of the ground height difference between non-motorized lanes and motorized lanes to carry out targeted construction of the modular system of barrier-free facilities. According to the current design of urban streets in China, the most widely used and direct street corner or some special sections of the pedestrian crossing barrier-free ramp. And for most municipal road construction, barrier-free ramp facilities modular system is even more essential.

According to the requirements of our country's barrier-free design code, it mainly puts forward the relevant requirements for the basic setting of barrier-free ramp, and ensures that the pedestrian system in the bridge, tunnel and three-dimensional crossing system in the urban road adopts barrierfree design. Specifically, the pavement should be designed at various junctions, entrances and exits, and these locations (pavement crossings and ends) will be provided with accessible edge stone ramps. Therefore, the modularization system of the prefabricated barrier-free ramp is mainly based on the design and construction of the edge stone ramp[1]. 


\section{Basic Design and Construction of Modular System of Barrier-free Ramp Facilities}

\subsection{Basic Design}

Generally speaking, there are three types of road widths on both sides of the city's municipal road, that is, the independent pavement (only for pedestrians), which adopts the pavement brick pavement structure; the non-mixed pavement, which is composed of non-motorized vehicles and pedestrians, is generally made of asphalt concrete pavement; and the non-motorized carriageway and pavement co-planar structure, which constructs the independent passage space between the non-motorized carriageway and the pedestrian passage, which is made of asphalt concrete and brick pavement, and the other parts are connected by the full-lying side stone. The above three types of cross-sections will set up special barrier-free access to form the final complete modular system of barrier-free ramp facilities, but the existing independent pavement barrier-free ramp facilities modular system has great drawbacks in the construction[2].

\subsection{General Construction Situation}

In the pavement construction process, will generally use a variety of materials and sizes of materials, including sintered brick, granite and concrete brick and so on. Combined with the past design and construction conditions, barrier-free ramps will be used in the construction of walking brick for special paving. And in the concrete construction process will first pass the pavement foundation to adjust the ramp height effectively, then the normal pavement surface layer, guarantees the ramp position to have the arc and the irregular edge angle, but the brick body position generally carries on the effective cutting according to the ramp size, finally splices the pavement successfully. In construction, we should strictly follow the ramp design form, such as the ramp design form according to the width of the ramp, slope and so on, to ensure the design of different types and sizes of ramps in the same grade road, so as to form a complete modular system of barrier-free ramp facilities later. In construction, the traditional bottleneck problems in pavement design and construction should be reduced, such as loose pavement brick rupture, dark spot, breakage and so on. Only in this way can the system be perfected and the ramp design effect be optimized [3].

\section{Research on Design and Application of Modular System for Assembly-type Barrier-free Ramp Facilities}

\subsection{Design Research Ideas}

The key to the modularization system of assembly barrier-free ramp design is splicing and module size design, which mainly aims at the different pavement structure form, material and thickness, etc., to ensure that the design research of facility modularization system is in place. For example, in whose process the standard specification of the ramp module is optimized to ensure that its design size reaches an integer multiple of the walking brick. Generally speaking, one-sided ramp module and three-sided ramp module can be selected for specific design. In particular, the ramp width can be arbitrarily adjusted by assembly, and the ramp module design includes two kinds of modules, the end and the middle, in which the end module mainly connects the sidewalk standard section, and the middle module can directly participate in the adjustment of the ramp width. Of course, it is also necessary to make a specific analysis of the specific problems according to the different use habits of different places, according to the different use habits and construction conditions of the pavement brick classification and size statistics, finally design a complete set of assembly barrier-free ramp module system to optimize some reconstruction roads. On the whole, the assembly splicing method is more time-saving and labor-saving, and its later maintenance and management is easier.[4].

All the modular materials in the modular system of prefabricated barrier-free ramp facilities are made of concrete, which can be produced in large quantities after modeling, which is called concrete preform. The advantages of the preform are that it has good anti-salt anticorrosion, beautiful exterior shape, convenient use and economical and durable value. It has been gradually 
applied in the municipal road engineering project, and its application value is very high[5].

\subsection{Design Applications}

In the process of specific design and application, we should strictly follow the relevant standard to investigate and analyze the construction of urban roads, especially the classification of pavement structure and related size statistics, the pavement surface mainly includes three kinds: concrete slab, sintered brick and granite slab. The three types of surface materials are varied in form, including $30 \times 30 \mathrm{~cm}, 40 \times 40 \mathrm{~cm}, 50 \times 50 \mathrm{~cm}, 50 \times 25 \mathrm{~cm}$ and so on, while the size of the trail brick is designed to be $10 \mathrm{x} 20 \mathrm{~cm}$. Finally, the side stone width of the road is $18 \mathrm{~cm}$, and the exposed height is $20 \mathrm{~cm}$. Because the road is designed with a modular system of prefabricated barrier-free ramp facilities, its ramp types include one-sided ramp and three-sided ramp, as shown in Figure 1[6].

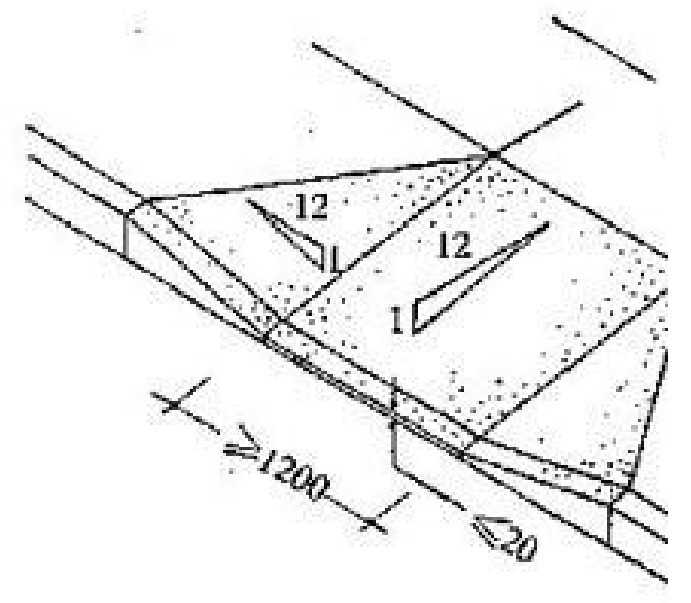

Figure 1 Three sidewalk ramp layout

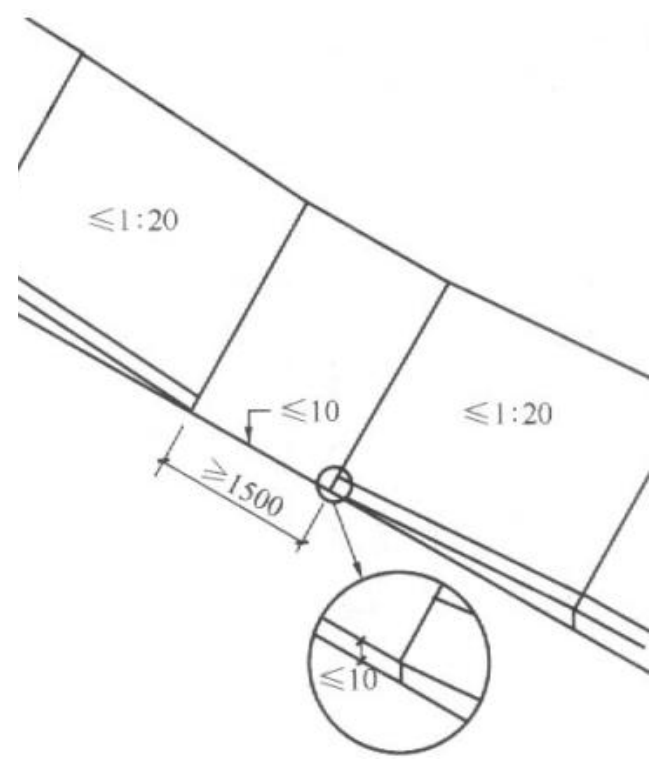

Figure 2 Sidewalk ramp graphic design

In combination with the design of the dimensions in the above drawing, the design plane size should also correspond to the depth of the design ramp in order to effectively adapt to the pavement of different widths, keep the slope height difference of the end module around $20 \mathrm{~cm}$, and ensure that the exposed height of the edge potential can be naturally transitioned to the underlying height. At the same time, to ensure the perfect splicing of the ramp module, fully consider the blind road design on the sidewalk in place, to ensure that the blind road connection is set in place in the ramp position, and to add the blind road layout module in the middle module position to ensure that it is connected with the standard road section, and to do a good job of module construction classification. 
In the construction of unobstructed need to produce 4 kinds of end module, form 8 kinds of standard ramp module type, based on the splicing combination form of different width, type of ramp targeted design and analysis, continuously optimize the rich module type and assembly mode, as shown in figure 3.

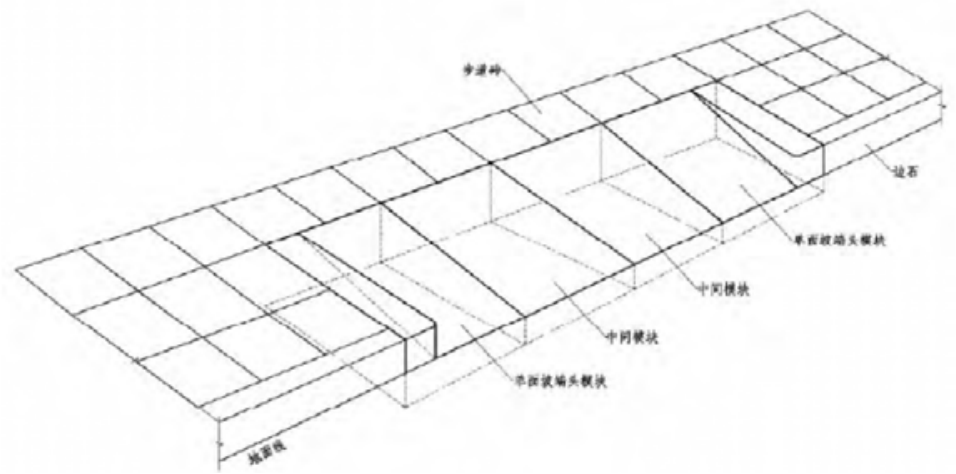

Figure 3 Chematic diagram of the design of the fabricated barrier-free ramp

In addition to the above design and construction methods, but also based on other conditions targeted treatment. For example, special design analysis should be carried out for some people's non-mixed pavement and non-conformities. For different areas of the city, the design of the prefabricated barrier-free ramp should adopt the fixed size and the fixed form, grasp the actual size of the section according to the requirements of the code, and optimize the size and type of the walkway brick according to the local actual situation, so as to ensure that it can match well with the walkway brick[7].

\section{Conclusion}

The modular system of prefabricated barrier-free facilities contains many kinds of design applications. For example, this paper discusses in detail the design and application system of prefabricated barrier-free sidewalk ramp in municipal road engineering project. It hopes to change the conventional design structure in traditional pavement barrier-free ramp and adopt concrete material module to carry out the whole process assembly construction. The assembly construction method has fundamentally changed the traditional design and construction technology, which is very beneficial to the optimization of municipal road construction at the present stage, and has created good social benefits for the development of urbanization, and the prospect of development is very worth looking forward to, adding color to the overall development of the city.

\section{Acknowledgements}

2019 Jiangsu province construction system science and technology project. The study on Building system of Low-rise assembly residential of Rural. (2019ZD001172)

\section{References}

[1] Chen, Di. A study on the design of an assembly barrier-free ramp. Journal of Liaoning Provincial College of Communications, no. 6, pp. 8-10, 2014.

[2] Foshan Institute of Science and Technology, South China University of Technology. An adjustable fabricated barrier-free ramp: CN201721684018.3, 2018.

[3] China Construction eighth Engineering Bureau Co., Ltd. Fast socket assembly accessible ramp: CN201320723435.X, 2014.

[4] Rodaway. Large span door bearing frame for assembly construction: CN201811333373, 2019.

[5] Song, Guohui., Wu, Jing., Song, Xihui. Assembly type barrier-free flat slope access device: 
CN201320828534.4, 2014.

[6] China Construction 8th Engineering Bureau Ltd. Steel lock assembly barrier-free ramp: CN201320727374.4, 2014.

[7] Chongqing Yijian Construction Group Co., Ltd. Assembly Support for Construction Engineering: CN201410298270.5, 2014. 